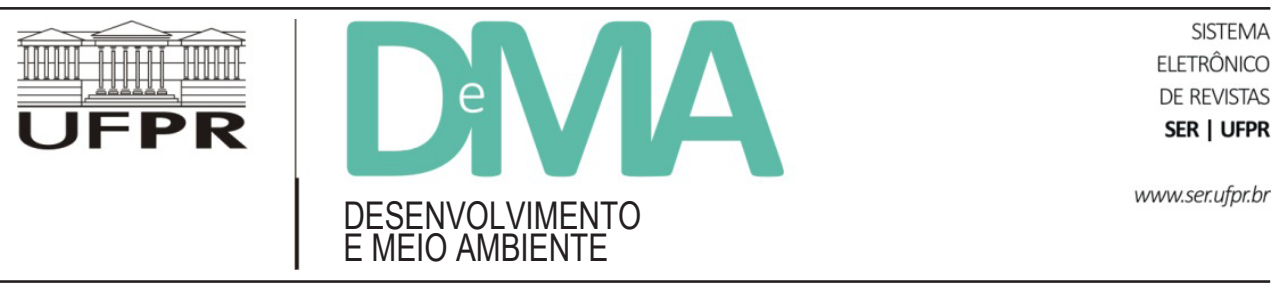

\title{
Efeitos ecológicos da exploração de produtos florestais não madeireiros: uma revisão sistemática
}

\section{Ecological Effects of Non-Timber Forest Products Harvest and Trade: a Systematic Review}

\author{
Alice Dantas BRITES ${ }^{1 *}$, Carla MORSELLO ${ }^{1}$ \\ ${ }^{1}$ Universidade de São Paulo (USP), São Paulo, SP, Brasil. \\ ${ }^{*}$ E-mail de contato: alicebrites@gmail.com
}

Artigo recebido em 16 de novembro de 2015, versão final aceita em 23 de abril de 2016.

RESUMO: A comercialização de produtos florestais não madeireiros (PFNM) é considerada estratégia que combina o desenvolvimento socioeconômico com baixo impacto ambiental. Porém, evidências demonstram que a exploração desses recursos pode provocar efeitos ecológicos negativos. Este estudo identificou os efeitos mais frequentes por meio de revisão sistemática de estudos empíricos, previamente submetidos a quatro critérios de inclusão e depois classificados conforme o tipo de PFNM explorado e os efeitos da exploração sobre 15 parâmetros em diferentes escalas biológicas. Os resultados indicaram que efeitos ecológicos negativos são frequentes, especialmente para a extração de cascas e folhas em grandes volumes, embora no curto prazo os efeitos possam ser nulos ou positivos. Os parâmetros mais frequentemente afetados foram: tamanho populacional; alterações nas partes vegetais; distribuição de classes de tamanho; probabilidade média de sobrevivência e riqueza de espécies. A constatação de que a atividade pode provocar efeitos ecológicos significativos evidencia a necessidade de sistemas de monitoramento e manejo.

Palavras-chave: recursos florestais; efeitos ecológicos; extrativismo; revisão.

ABSTRACT: Trade of non-timber forest products (NTFP) is a strategy that combines socioeconomic development with activities of low environmental impact. However, evidence suggests that harvesting these resources can produce negative ecological effects. This article identified the most common effects through a systematic literature review of empirical studies. Articles were previously evaluated against four criteria of inclusion, and were then classified according to the plant part harvested and the effects on 15 parameters at different levels of the biological organization. The results indicated that negative ecological effects are common, especially when harvesting barks and leaves in large volumes, although null and positive effects may also be observed. The parameters most frequently affected were population size; modifications in plant parts; age structure; probability of survival, and species richness. The evidence that NTFP harvest can cause significant ecological impacts calls for a need of monitoring and management systems.

Keywords: forest products; ecological effects; harvest; review. 


\section{Introdução}

Produtos florestais não madeireiros (PFNM), aqui definidos como originários de plantas ou fungos, como cascas, resinas, fibras, sementes, frutos e corpos de frutificação, têm sido, desde os anos 1980, foco de interesse de organizações governamentais e não governamentais. Para elas, a coleta desses produtos em ambientes naturais como florestas, assim como sua comercialização, deveriam ser incentivadas, pois promoveriam concomitantemente a conservação da biodiversidade e a melhoria da qualidade de vida dos habitantes locais (Shackleton et al., 2011). O entusiasmo com esta ideia baseou-se na premissa de adequação desses produtos em termos de desenvolvimento socioeconômico e conservação da biodiversidade (Kusters et al., 2006).

Pela vertente do desenvolvimento, os PFNM já são coletados por centenas de milhares de pessoas no mundo para uso próprio como alimentos, produtos medicinais e matérias-primas para a confecção de artesanato, ferramentas de trabalho e construção civil (Shackleton et al., 2011; Ticktin \& Shackleton, 2011). Como a coleta de PFNM não tem alta demanda de capital financeiro ou de infraestrutura, sua exploração é acessível a grupos menos favorecidos (Ros-Tonen \& Wiersum, 2005), que, além de utilizarem os PFNM para consumo, frequentemente dependem deles como única fonte de renda monetária (Ruiz-Pérez et al., 2004). Portanto, seu importante papel na economia cotidiana de habitantes de áreas naturais e como fonte de recursos de salvaguarda em momentos de crise (Shackleton \& Shackleton, 2004) faz desses produtos uma escolha considerada adequada para a promoção do desenvolvimento local.

Pelo lado da conservação da biodiversidade, os PFNM são considerados favoráveis, pois sua exploração incentiva os habitantes a manterem os ecossistemas naturais em pé, pois deles derivam recursos financeiros (Sills et al., 2006). Ademais, explorá-los usualmente gera menores impactos ambientais que outros usos da terra, como agricultura, pecuária e exploração madeireira (Peters, 1994).

Todavia, como esperado para qualquer atividade antrópica, especialmente em escala comercial, o extrativismo de PFNM gera impactos ecológicos não negligenciáveis, os quais dependem da forma de vida, parte da planta explorada, época e quantidade extraída, além das técnicas de extração e manejo empregadas (Ticktin, 2004). Por sua vez, os efeitos ecológicos podem ser observados no nível dos indivíduos (e.g., taxa de crescimento) e das populações (e.g., taxas de mortalidade e recrutamento) da espécie explorada, ou na comunidade biótica (e.g., redução de recursos alimentícios para fauna) e nos ecossistemas de origem (e.g., redução de nutrientes no solo) (Kusters, 2009).

Em suma, os possíveis efeitos do extrativismo de PFNM são variados e devem ser avaliados considerando diferentes níveis ecológicos e as partes extraídas (Ticktin, 2004). Este artigo tem, portanto, o objetivo de apresentar uma revisão sistemática do conhecimento atual sobre os efeitos ambientais do extrativismo de PFNM, como forma de subsidiar a escolha de indicadores a serem monitorados para garantir a sustentabilidade do manejo de PFNM.

\section{Metodologia}

O levantamento de dados dividiu-se em duas etapas. Inicialmente, foi realizado um levantamento dos possíveis efeitos ecológicos da comercialização de PFNM por meio de uma revisão não sistemática da literatura. Esta etapa teve como objetivo levantar artigos de revisão ou compilações acerca do tema. A partir deste levantamento inicial, foram: (i) identificadas as hipóteses existentes acerca dos efeitos 
da exploração de PFNM nos diversos níveis organização biológica e (ii) levantados os parâmetros ecológicos a serem utilizados na etapa seguinte para a classificação das evidências científicas.

A segunda etapa, em contraste com a primeira, consistiu em revisão sistemática da literatura. Essa forma de revisão estabelece previamente e torna explícitos os critérios utilizados na busca e na seleção de publicações, resultando em levantamento abrangente e rigoroso do tópico pesquisado, que evita enviesamentos e aumenta a confiabilidade da discussão (Akobeng, 2005). O levantamento foi realizado nas seguintes bases de dados: Scientific Electronic Library Online (ScieELO); Web of Science (WoS); Center for International Forestry Research (CIFOR) e Digital Library of the Commons (DLC). As duas últimas instituições possuem vastos acervos de publicações referentes à conservação e ao uso de recursos naturais e, por este motivo, foram incluídas nas bases de dados utilizadas. Em cada uma dessas bases, o levantamento foi realizado por meio dos mecanismos de pesquisa presentes, adotando como padrão inicial as mesmas palavras-chave (Figura 1). Em seguida, para as buscas que resultaram em grande número de publicações ( $n \geq 60$ ), foram utilizados mecanismos de refinamento dos resultados com a inclusão de palavras-chave mais específicas. As publicações resultantes foram posteriormente avaliadas a partir dos seguintes critérios de inclusão: (i) tratavam de produtos florestais derivados de plantas ou fungos, excluindo produtos de origem animal; (ii) apresentaram análise em floresta natural, excluindo ecossistemas antrópicos; (iii) apresentaram dados empíricos primários, excluindo revisões bibliográficas; e (iv) especificavam a parte, ou partes, da planta explorada. Referências secundárias citadas nestes artigos foram incluídas e submetidas aos mesmos critérios.
Para as publicações que permaneceram na amostra, foram levantados o tipo de PFNM explorado (Tabela 1) e os possíveis efeitos sobre parâmetros ecológicos dos seguintes níveis de organização biológica: população da espécie explorada, comunidade, ecossistema e paisagem nos quais ocorre a exploração. Os parâmetros adotados em cada um desses níveis (total de 15; Tabela 2) foram compilados a partir do levantamento não sistemático e preliminar de artigos. Os efeitos sobre cada um desses parâmetros descritos nos artigos da amostra foram classificados como: (i) nulos, quando não foram observadas alterações; (ii) negativos, quando prejudicaram os indivíduos explorados, diminuíram taxas ou parâmetros das populações, comunidades ou ecossistemas com implicações negativas à conservação; e (iii) positivos, quando favoreceram os indivíduos explorados, aumentaram taxas ou parâmetros da população, comunidade ou ecossistema.

TABELA 1 - Classificação quanto ao tipo de PFNM explorado.

\begin{tabular}{|c|c|}
\hline Tipo de PFNM & Definição \\
\hline Flor & Flores ou partes da flor \\
\hline Semente & $\begin{array}{l}\text { Sementes ou partes da semente (casca, } \\
\text { endosperma, embrião) }\end{array}$ \\
\hline Fruto & $\begin{array}{l}\text { Frutos ou partes do fruto (epicarpo, } \\
\text { mesocarpo e endocarpo) }\end{array}$ \\
\hline Folha & $\begin{array}{l}\text { Folhas ou partes extraídas de folha (por } \\
\text { ex. fibras) }\end{array}$ \\
\hline Caule & $\begin{array}{l}\text { Caules ou partes extraídas de caules } \\
\text { (por ex. fibras) }\end{array}$ \\
\hline Casca & Córtex caulinar \\
\hline Raiz & Raiz ou partes da raiz \\
\hline $\begin{array}{l}\text { Composto } \\
\text { secundário }\end{array}$ & $\begin{array}{l}\text { Produtos oriundos do metabolismo } \\
\text { secundário (por ex. resina, goma, látex, } \\
\text { óleo) }\end{array}$ \\
\hline Planta inteira & $\begin{array}{l}\text { Plantas que são utilizadas inteiras em } \\
\text { sua forma jovem ou adulta }\end{array}$ \\
\hline $\begin{array}{l}\text { Corpo de } \\
\text { frutificação }\end{array}$ & $\begin{array}{l}\text { Estrutura reprodutiva macroscópica de } \\
\text { fungos }\end{array}$ \\
\hline
\end{tabular}


Diversos artigos trazem evidências científicas de efeitos ecológicos sobre mais de uma espécie, resultantes da coleta de mais de uma parte vegetal ou dos resultados da extração sobre mais de um parâmetro ecológico. Portanto, o número total de casos $(\mathrm{n}=182)$ é maior do que o número total de publicações $(\mathrm{n}=84)$.

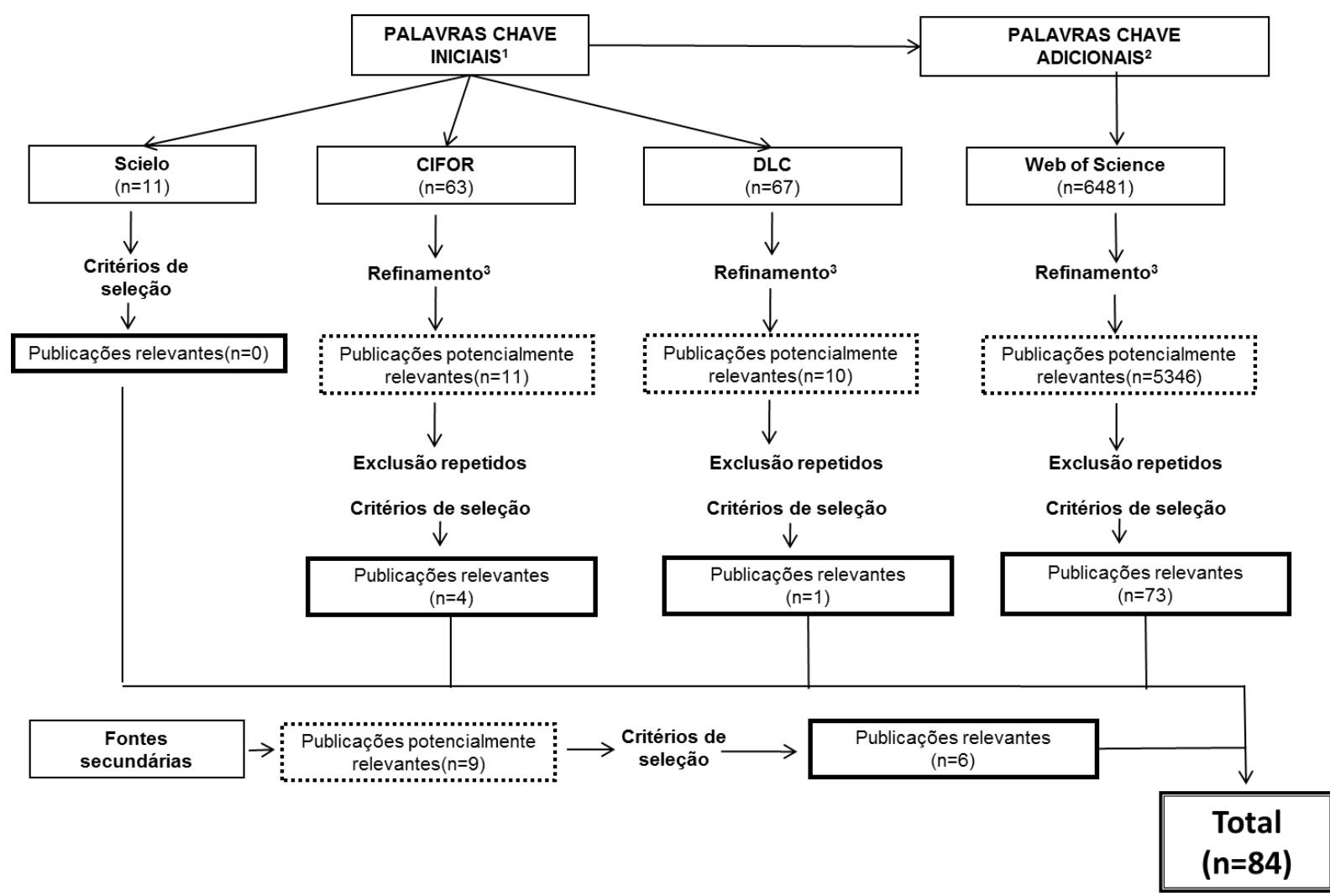

FIGURA 1 - Representação esquemática das estratégias de busca utilizadas na revisão sistemática da literatura.

1 "non timber", "nontimber", "nonwood", "non-timber", "non wood" e "non- wood"

${ }^{2} \mathrm{TS}=$ (plant AND Forest) AND TS $=($ commer* AND harvest* $)$; TS $=($ seed AND Forest $)$ AND TS $=($ commer* AND harvest $*)$ TS $=($ fruit AND Forest $)$ AND TS $=($ commer* AND harvest* $) ;$ TS $=($ leaf OR leaves $)$ AND TS $=($ forest $)$ AND TS $=($ commer* AND harvest* $)$; TS $=($ flower* AND Forest $)$ AND TS $=($ commer* AND harvest* $)$; TS $=($ bark AND Forest $)$ AND TS $=($ commer* AND harvest $*$; TS $=($ root* AND Forest $)$ AND TS $=($ commer* AND harvest* $) ;$ TS $=($ stem* AND Forest $)$ AND TS $=($ commer* AND harvest* $) ;$ TS $=($ branch* AND Forest $)$ AND TS $=($ commer* AND harvest $*) ; \mathrm{TS}=($ apical AND meristem $)$ AND TS $=($ forest $)$ AND TS $=($ commer* AND harvest* $) ;$ TS $=($ resin* AND forest $)$ AND TS $=($ commer* AND harvest* $)$

${ }^{3}$ Buscas refinadas por meio das palavras: "ecolog* or impact* or effect* or issue* or outcome* or success* or threat* or harvest* or exploitation*" 
TABELA 2 - Parâmetros ecológicos avaliados e possíveis efeitos provocados pela exploração de PFNM.

\begin{tabular}{|c|c|c|c|}
\hline Nível & Parâmetro & Efeito & Referência \\
\hline \multirow{9}{*}{ 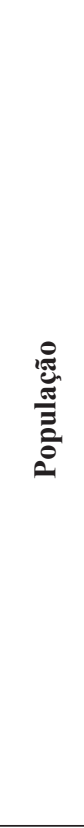 } & Taxa de crescimento & $\begin{array}{l}\text { Aumento ou diminuição da taxa de crescimento (altera produção ou } \\
\text { incorporação de biomassa do corpo do vegetal) }\end{array}$ & $(\mathrm{C} ; \mathrm{HB} ; \mathrm{T})$ \\
\hline & Sobrevivência & Alterações na probabilidade de sobrevivência do espécime explorado & $(\mathrm{C} ; \mathrm{HB} ; \mathrm{P} ; \mathrm{T})$ \\
\hline & Reprodução & $\begin{array}{l}\text { Aumento ou diminuição da taxa de reprodução sexuada ou vegetativa, por } \\
\text { meio, por exemplo, de alterações na produção de frutos ou sementes }\end{array}$ & $(\mathrm{C} ; \mathrm{HB} ; \mathrm{P} ; \mathrm{T})$ \\
\hline & Parte vegetal & $\begin{array}{l}\text { Alterações nos órgãos reprodutivos ou vegetativos da planta, levando } \\
\text { à produção, por exemplo, de órgãos maiores ou menores, em maior ou } \\
\text { menor quantidade, alterações em processos fisiológicos e alterações no } \\
\text { vigor }\end{array}$ & $(\mathrm{C} ; \mathrm{P})$ \\
\hline & Estrutura genética & Modificação das frequências gênicas & $(\mathrm{HB} ; \mathrm{P})$ \\
\hline & Estrutura espacial & Restrição ou ampliação da distribuição espacial local & $(\mathrm{K})$ \\
\hline & Classes de tamanho & $\begin{array}{l}\text { Alteração na estrutura etária natural, com maior ou menor número de } \\
\text { indivíduos em uma das classes etárias }\end{array}$ & $(\mathrm{HB} ; \mathrm{K} ; \mathrm{P})$ \\
\hline & Razão sexual & Alterações na razão sexual em espécies dioicas & $(\mathrm{K})$ \\
\hline & Tamanho & $\begin{array}{l}\text { Aumento ou diminuição do número de indivíduos da população } \\
\text { provocado, por exemplo, por alterações na taxa de dispersão de sementes, } \\
\text { no sucesso de estabelecimento de novas plântulas, na reprodução ou na } \\
\text { probabilidade de sobrevivência. }\end{array}$ & $(\mathrm{HB} ; \mathrm{K}, \mathrm{P})$ \\
\hline \multirow{3}{*}{ 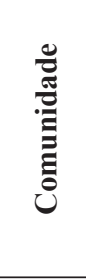 } & Composição & Aumento ou diminuição da riqueza de espécies & $(\mathrm{T})$ \\
\hline & $\begin{array}{l}\text { Interação planta- } \\
\text { planta }\end{array}$ & $\begin{array}{l}\text { Alterações nas interações entre plantas de uma mesma comunidade, por } \\
\text { exemplo, na competição interespecífica }\end{array}$ & $(\mathrm{T})$ \\
\hline & $\begin{array}{l}\text { Interação planta- } \\
\text { animal }\end{array}$ & $\begin{array}{l}\text { Alterações nas interações entre plantas e animais de uma mesma } \\
\text { comunidade, por exemplo, relações de predação, parasitismo, polinização, } \\
\text { dispersão de sementes, recursos disponíveis para frugívoros }\end{array}$ & $(\mathrm{HB} ; \mathrm{P} ; \mathrm{T})$ \\
\hline \multirow{2}{*}{ 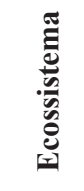 } & Ciclos energéticos & Alterações no ciclo energético, por exemplo, alterações na teia alimentar & $(\mathrm{K})$ \\
\hline & Parâmetros físicos & $\begin{array}{l}\text { Alterações nos parâmetros físicos, por exemplo, altera concentração de } \\
\text { nutrientes no solo, luminosidade }\end{array}$ & $(\mathrm{HB} ; \mathrm{K} ; \mathrm{T})$ \\
\hline 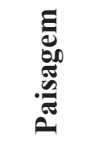 & Corredor ecológico & $\begin{array}{l}\text { Alterações na paisagem local ou regional, mesmo que indiretamente } \\
\text { (abertura de trilhas ou estradas) }\end{array}$ & $(\mathrm{K})$ \\
\hline
\end{tabular}

Notas: (1) Cunningham, 2001 (C); Hall \& Bawa, 1993 (HB); Kusters, 2009 (K); Peters, 1994 (P); Ticktin, 2004 (T). 


\section{Resultados e discussão}

\subsection{Distribuição geográfica dos estudos}

Dos 84 estudos identificados, a grande maioria refere-se à África, América do Sul e América do Norte (Figura 2). Os estudos, portanto, negligenciam a Europa, onde a extração de PFNM é substancial (Fao, 2005). Também existem menos estudos do que o esperado para a Ásia, dada a importância dos PFNM neste continente. Embora exudatos (óleos e resinas) asiáticos representem a quase totalidade $(95,5 \%)$ da quantidade destes produtos comercializados mundialmente, há apenas uma avaliação da extração deste PNFM para o continente (Varghese \& Ticktin, 2008). Ressalta-se, contudo, que as estatísticas globais do comércio de PFNM são imprecisas, pois esses produtos são frequentemente invisíveis à economia formal. As estatísticas também não consideram se o produto é extraído de ecossistemas naturais ou antrópicos, enquanto o presente artigo inclui apenas os primeiros, razão que contribui para as diferenças encontradas.

A distribuição dos estudos é desigual não apenas entre os continentes, mas também dentro destes. Para a África, a maioria dos estudos provém da África do Sul e Benin em Florestas Tropicais Úmidas ou Florestas Tropicais Semiáridas, enquanto o resto do continente é negligenciado. Na América do Sul, as avaliações concentram-se nas Florestas Tropicais Úmidas da Amazônia, enquanto para as Florestas Tropicais da Mata Atlântica, Florestas Tropicais Semiáridas e Florestas Temperadas Úmidas existem pouquíssimas publicações. Na Ásia, apesar do maior volume de extração de PFNM ser indicado para a China (Fao, 2005), foi identificado apenas um estudo para o país (Chen, 2004), enquanto a maioria das evidências provém de Florestas Tropicais Úmi- das ou Savanas da Índia. Para outros países asiáticos com grande volume de extração, como Coréia do Sul e Paquistão (Fao, 2005), não há estudos. Na Europa, continente com grande volume de extração de PFNM, foi identificado apenas um trabalho (Egli et al., 2006), realizado em Floresta Temperada Úmida. São, portanto, ausentes áreas com grandes volumes de extração, como as Florestas Boreais Úmidas da Finlândia, Suécia e República Tcheca (Fao, 2005). Na América do Norte, a grande maioria das evidências provém de Florestas Tropicais Úmidas do México e, parte menor, de Florestas Temperadas Úmidas dos Estados Unidos. Para a Oceania, somente um estudo em Floresta Tropical Úmida foi identificado (Widayati \& Carlisle, 2012), desconsiderando as Florestas Tropicais Semiáridas e as Florestas Temperadas Úmidas e Semiáridas.

\subsection{Características dos PFNM estudados}

Um total de 76 espécies de 41 famílias foi avaliado, sendo 57 arbóreas, 12 herbáceas, 2 arbustivas e 2 lianas. Pteridófitas, liquens e fungos são formas de vida pouco estudadas, cada qual com uma espécie avaliada nos estudos amostrados. A família das palmeiras, Arecaceae, teve mais espécies estudadas (13), seguida pela família Fabaceae (6 espécies). Euphorbiaceae, Meliaceae e Anacardiaceae foram cada qual representadas por quatro espécies.

A distribuição dos estudos em relação à parte da planta explorada também é desbalanceada. As partes mais estudadas são folhas $(24 \%, n=182$ evidências) e cascas (19\%), embora caules e frutos também apareçam nas avaliações (13 e 11\%, respectivamente). A análise de raízes e corpos de frutificação de fungos é, todavia, rara $(0,4$ e $0,2 \%$, respectivamente). 


\subsection{Escalas de avaliação de impacto}

A maioria das análises $(92 \%, \mathrm{n}=182)$ investigou efeitos do extrativismo sobre as populações exploradas (Figura 2). A ênfase nesta escala era esperada, pois neste nível os efeitos são diretos e imediatos, bem como o pequeno tamanho populacional é considerado o principal fator de vulnerabilidade de espécies (Purvis et al., 2000). Por sua vez, as poucas evidências na escala de comunidade e a ausência total para ecossistemas e paisagens limitam as inferências possíveis nesses níveis. Como não existem evidências empíricas diretas, é impossível concluir se a extração causa mudanças em parâmetros físicos ou no papel das áreas como corredores ecológicos, como previamente argumentado (Hall \& Bawa, 1993; Kusters, 2009).

\subsection{Tipos de efeitos da coleta de PFNM}

Cerca de $64 \%(n=182)$ das avaliações sugeriram que a exploração de PFNM produz efeitos ecológicos negativos nos parâmetros avaliados (Tabela 2). Todavia, parte substancial dos casos (32\%) não detectou efeitos do extrativismo, em especial para a coleta de folhas, enquanto $4 \%$ dos estudos identificaram efeitos positivos, ao menos durante o curto período estudado.

$\mathrm{O}$ extrativismo de folhas e cascas frequentemente causou efeitos negativos $(23 \%$ e $22 \%$, respectivamente). Embora em pequeno número, estas partes vegetais também figuram naquelas com efeitos nulos e positivos. Em parte, os resultados contrastam com a literatura científica, pois hipóteses prévias indicavam que a coleta de partes reprodu-

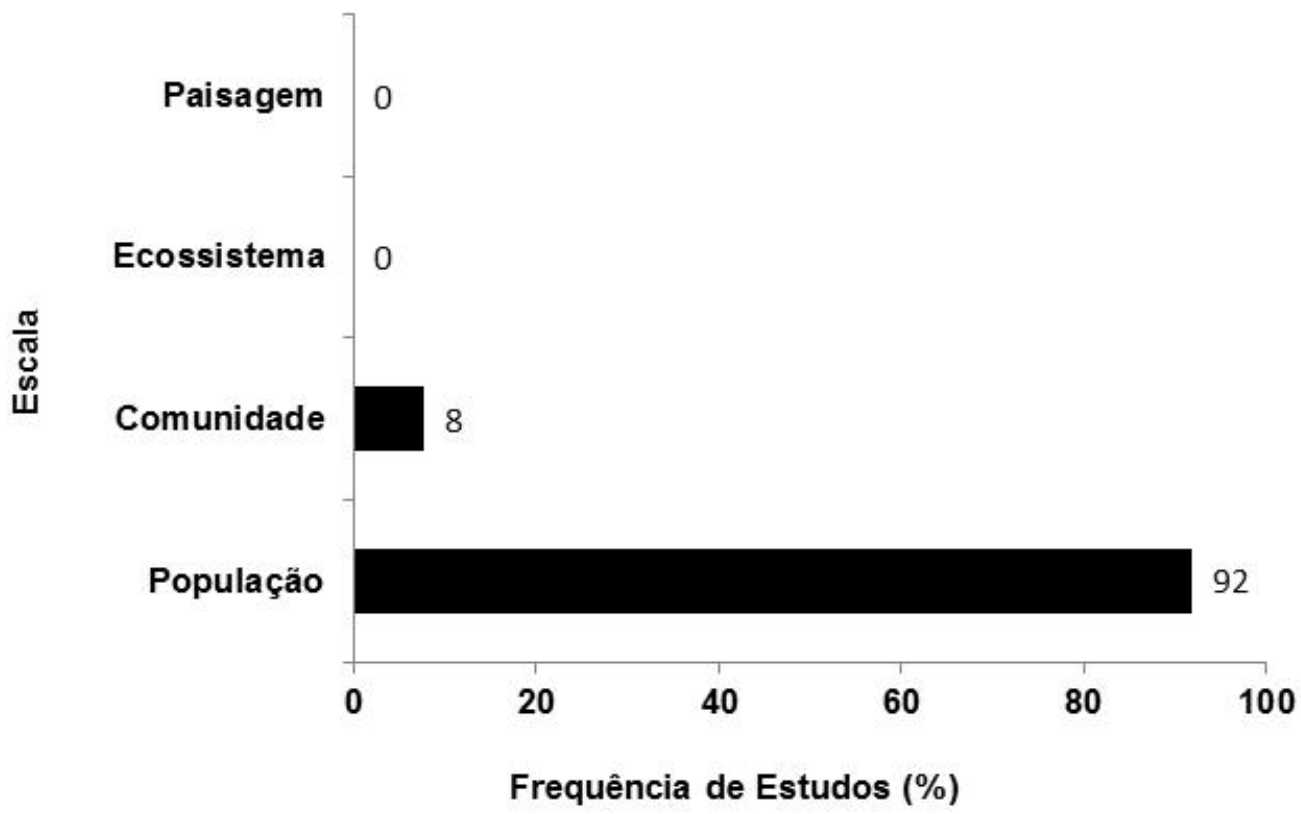

FIGURA 2 - Frequência de casos por escala de organização ecológica $(\mathrm{n}=182)$. 
tivas (flores, frutos e sementes) e de folhas provocaria efeitos ecológicos menos severos ou menos frequentes, quando comparada à coleta de partes vegetais que podem levar a planta à morte (i.e., casca e raiz) (Hall \& Bawa, 1993; Cunningham, 2001). Porém, quando sistematizadas, as evidências sugerem que os efeitos negativos decorrentes da coleta de folhas são comuns, ao menos quando coletadas em grandes quantidades, ou quando a extração de folhas está associada à coleta de outras partes vegetativas (e.g., Endress et al., 2006).

\subsection{Efeitos sobre a população explorada}

A análise incluiu 168 evidências dos efeitos na escala de população, distribuídos entre nove parâmetros considerados: tamanho populacional, distribuição de classes de tamanho, partes vegetais, taxa de crescimento dos indivíduos, probabilidade de sobrevivência, reprodução, estrutura genética da população, distribuição espacial e razão sexual.

\subsubsection{Tamanho populacional}

O efeito da extração sobre o tamanho da população explorada foi o aspecto mais frequentemente avaliado nesta escala $(32 \%, \mathrm{n}=168)$, com $58 \%$ das evidências indicando efeitos negativos, embora porcentagem considerável (42\%) não tenha observado alterações (Figura 3).

Em diversos estudos, a coleta de partes reprodutivas como frutos (e.g., Shackleton et al., 2005), sementes (Raimondo \& Donaldson, 2003) e corpos de frutificação de fungos (Chen, 2004) causou declínios populacionais. Padrão similar foi observado com a coleta de plantas inteiras de diversas briófitas (Peck, 2006) ou de uma herbácea (Allium tricoccum Ait.) (Rock et al., 2004), mas também de partes vegetativas como raízes (Larsen, 2002; Plowden et al., 2003), compostos secundários (e.g. resinas) (Gebrehiwot et al., 2003), cascas (e.g., Gaoue \& Ticktin, 2008), caules (Ghimire et al., 2005) e folhas (quando a coleta retira a maior parte das folhas) (Endress et al., 2006).

Porém, também existem evidências de efeito nulo no tamanho populacional, observadas em estudos com coleta de partes reprodutivas (e.g., Emanuel et al., 2005), vegetativas (Pedersen, 1996; Zuidema et al., 2007) e corpo de frutificação de fungos (Marshall \& Newton, 2003).

Os resultados também mostram que formas de coleta que usualmente causam a morte da planta, como a coleta da planta inteira (comum em briófitas e herbáceas) ou da raiz e, às vezes, dos caules, estão frequentemente associadas a declínios populacionais. As poucas exceções parecem estar associadas a plantas capazes de se reproduzir vegetativamente (Ghimire et al., 2005). Para a coleta de outras partes vegetativas, como folhas e cascas, ou reprodutivas, os resultados são mistos. No caso das folhas, comumente extraídas de palmeiras, as evidências sugerem que, apesar dos efeitos negativos, certos níveis de extração, por vezes até elevados, podem ser tolerados, muito embora os efeitos demográficos sejam cumulativos e apenas observáveis no longo prazo (Endress et al., 2006). Também no caso de partes reprodutivas os resultados são variáveis e dependem de atributos diversos, como formas de manejo e extrativismo, ou história de vida da planta. Para espécies longevas, é difícil perceber os efeitos populacionais, a não ser que estudos monitorem as populações por prazos longos. Por exemplo, estudos de curto prazo com a extração de castanha-do-brasil (Bertholletia excelsa Humb. \& Bonpl.) indicaram que a coleta nos níveis usuais tem efeitos nulos (Zuidema, P. A. \& Boot, R. G. A., 2002). Em contraste, a comparação de populações com históricos 
diversos de extração e, portanto, incluindo variáveis de longo prazo, evidenciou que a coleta pode ser insustentável (Peres et al., 2003). Apesar de dados de longo prazo serem essenciais para entender certos padrões, apenas cerca de $10 \%$ dos estudos tinham esta abordagem até recentemente (Ticktin, 2004).

\subsubsection{Partes vegetais}

Em segundo lugar em número de estudos, há os efeitos do extrativismo sobre partes vegetais das plantas das populações exploradas $(23 \%, \mathrm{n}=168)$, a maioria dos quais (70\%) são negativos (Figura 3). Diversas espécies de árvores, por exemplo, apresentaram pouca ou mesmo nenhuma recuperação do córtex caulinar após a remoção da casca (e.g.,Delvaux, 2009). Já para a extração de folhas de diferentes espécies de palmeiras, foi observada redução na área média das folhas produzidas em anos subsequentes à exploração (e.g.,Endress, Gorchov, Peterson, et al., 2004). Efeito similar de redução do tamanho dos ramos foi observado também após a coleta das hastes e folhas da her-

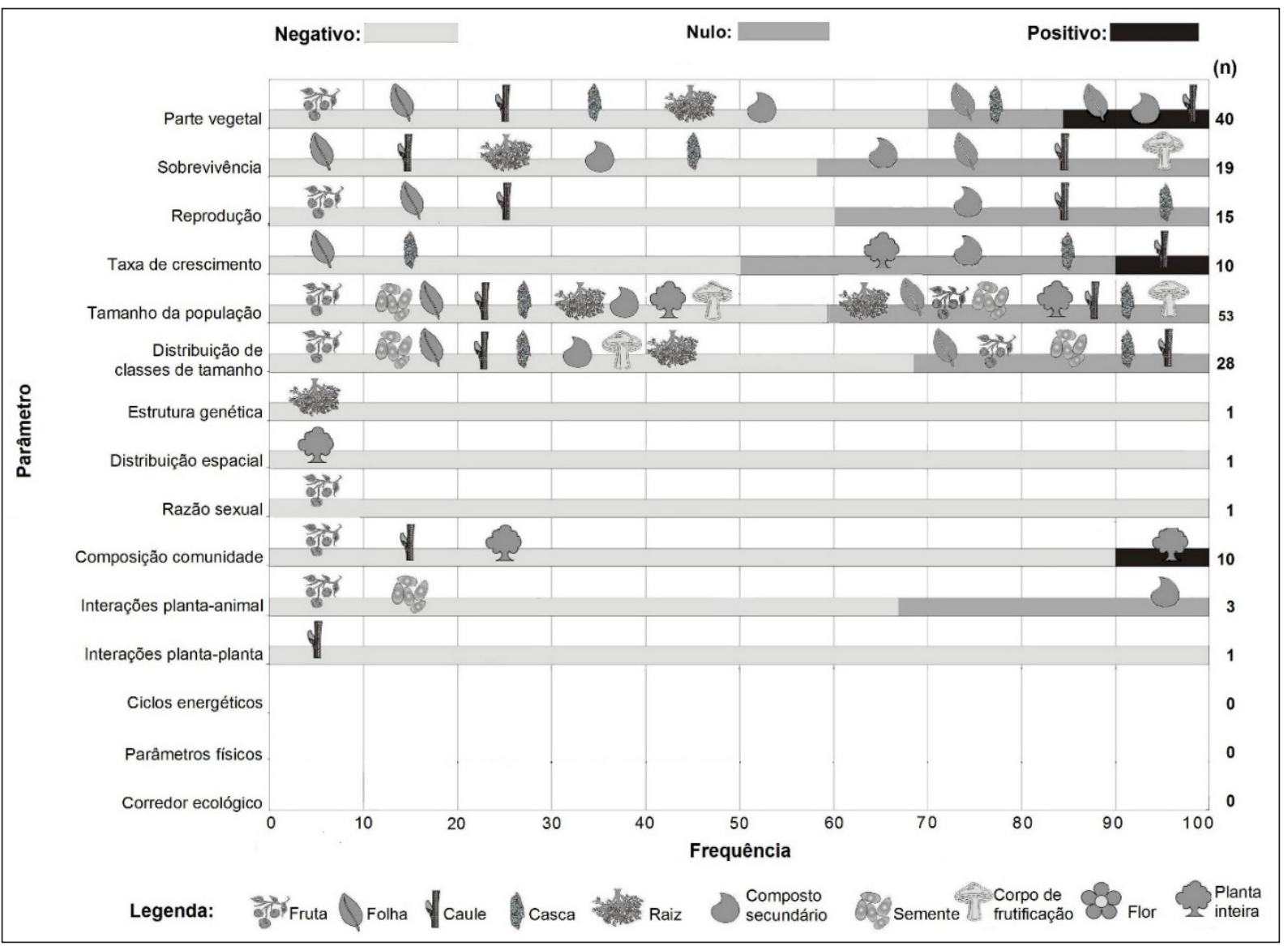

FIGURA 3 - Frequência dos efeitos ecológicos da exploração encontrados nos estudos considerados, segundo tipo de efeito e de PFNM explorado. Os parâmetros ecológicos avaliados estão no eixo esquerdo. As figuras sobre as barras indicam o tipo de PFNM para o qual as evidências foram encontradas $(n=182)$. 
bácea Hydrastis canadenses L., efeitos estes que variaram segundo a época em que a coleta ocorreu (Sanders \& Mcgraw, 2005). Para frutos, há apenas duas evidências, originadas de um mesmo estudo com árvores do gênero Phyllanthus (Phyllanthaceae). Neste, mostrou-se que a coleta de frutos em taxas altas ( $90 \%$ do total) causou menor produção média de frutos por árvore no ano subsequente, principalmente quando os ramos eram dobrados e cortados durante a extração (Sinha \& Bawa, 2002). Outro estudo observou a redução do comprimento e da biomassa médios das raízes de indivíduos da espécie medicinal Nardostachys grandiflora (D. Don) DC. submetidos à exploração por certo tempo, quando comparados a indivíduos de populações-controle nunca exploradas. Ou seja, a exploração alterou características dos indivíduos explorados, ocasionando efeitos populacionais de longo prazo (Larsen, 2002).

Os resultados apresentados concordam, portanto, com Cunningham (2001), que relatou que a exploração de partes vegetativas ou de frutos pode prejudicar o desenvolvimento normal das partes vegetais, por exemplo, devido ao redirecionamento de nutrientes para reparar partes danificadas pela coleta. Assim, a coleta de certas partes, como folhas, pode ter efeitos pequenos para o estoque de folhas em si, mas pode provocar redirecionamento de recursos para recomposição do tecido foliar que seriam alternativamente utilizados para a reprodução (Endress, Gorchov, Peterson, et al., 2004). Nestes casos, os efeitos observados no curto prazo podem ser pequenos, embora no longo prazo seja possível que os efeitos demográficos sejam significativos.

Ressalta-se também que, ao menos com os níveis de extração avaliados, cerca de $15 \%$ dos casos não encontraram evidências de alterações negativas em partes vegetais (e.g.,Pedersen, 1996), assim como há casos (15\%) em que a exploração produziu efeitos positivos nos indivíduos explorados, como maior produção de brotos (Siebert, 2000), rizomas ou raízes (Vázquez-Lopez et al., 2004). Esses resultados podem ser devidos a respostas compensatórias das plantas, como o redirecionamento de reservas energéticas para compensar perda de tecidos, ou podem refletir maiores taxas fotossintéticas em resposta a incrementos na luminosidade causados por aberturas no dossel (Niels et al., 2003). Respostas compensatórias são consideradas comuns, ao menos para herbáceas e palmeiras sujeitas ao extrativismo (Schmidt et al., 2011).

Os resultados dos estudos também evidenciam que a época (e.g., Sanders \& Mcgraw, 2005), taxa ou frequência de extração das partes vegetais (e.g., Endress et al., 2006) e os métodos de coleta (e.g.,Sinha \& Bawa, 2002) podem, dependendo da espécie, ter consequências diversas. No caso das estratégias de coleta, há técnicas mais ou menos impactantes. Por exemplo, embora algumas técnicas tenham por meta extrair apenas partes como folhas e cascas, valem-se da derrubada da planta. Outras dobram a planta ou os ramos no momento da coleta ao invés de chacoalhar e bater no caule, o que aumenta os efeitos negativos de longo prazo (Sinha \& Bawa, 2002). No caso da extração de cascas, a remoção de anéis inteiros do córtex caulinar, ao invés da extração parcial, provoca efeitos maiores (Guedje et al., 2003). Evidências como estas são usualmente levantadas por estudos experimentais, os quais mensuram por alguns anos os efeitos de diferentes manipulações. São, todavia, mais raros que estudos observacionais, nos quais costumam faltar informações sobre o volume e as técnicas de extrativismo. 


\subsubsection{Distribuição de classes de tamanho}

Dentre as avaliações dos efeitos da coleta sobre a proporção de diferentes classes de tamanho da população explorada $(17 \%, \mathrm{n}=168), 61 \%$ observaram efeitos negativos e 39\% não observaram alterações (Figura 3).

Para a coleta de frutos ou sementes (e.g., Raimondo \& Donaldson, 2003; Avocèvou-Ayisso et al., 2009), folhas (e.g.,Rodriguéz-Buriticá et al., 2005), caules (Raimondo \& Donaldson, 2003), cascas (Botha et al., 2004; De Oliveira et al., 2007) e o corpo de frutificação de fungos (Chen, 2004) foi observada, como esperado, a redução no número de indivíduos de ao menos uma das classes de tamanho da população. Em contraste, outros estudos com a coleta de sementes (Wadt et al., 2008), frutos (e.g., Ganesan \& Setty, 2004), caules (Griffiths et al., 2005) e flores (Marshall \& Newton, 2003) não observaram efeitos sobre a estrutura etária da população relacionados ao extrativismo (Zuidema, P.A. \& Boot, R.G.A., 2002).

Novamente, os efeitos variaram, podendo tanto indicar que a extração tem poucos efeitos, como também serem devidos a baixos níveis de extração ou à exploração recente, fator importante para espécies longevas. Esses fatores são muitas vezes negligenciados, pois poucos estudos avaliam os efeitos de níveis diversos de exploração e, ainda mais raramente, históricos diferentes de extração (e.g. Peres et al., 2003). No caso de espécies longevas, quando estudos experimentais podem ser inviáveis, o acompanhamento do histórico de extração e comparação da demografia de diferentes populações é importante. Níveis de exploração elevados e recorrentes podem reduzir consideravelmente o recrutamento de jovens e a sobrevivência de adultos, produzindo colapso demográfico no longo prazo, embora apenas estudos mais abrangentes possam demonstrar este efeito.

\subsubsection{Probabilidade de sobrevivência}

Os efeitos da coleta sobre a taxa de sobrevivência dos indivíduos das populações exploradas ocupam o quarto lugar com $11 \%(n=168)$ do total das avaliações no nível de população. Grande parte destas identificou efeitos negativos (58\%), embora percentual considerável (42\%) não observou alterações com os níveis de exploração ou técnicas de coleta considerados (Figura 3).

No caso da redução da taxa, foi observada com a exploração de diferentes partes de plantas. A coleta de cascas, especialmente de toda a circunferência da planta, levou à redução na probabilidade de sobrevivência nas espécies medicinais arbóreas (Cocks \& Dold, 2004). Efeito similar ocorreu com a coleta de folhas de palmeira, quando estas sofrem grande defoliação da copa (Chamaedorea radicalis Mart.), quer seja apenas uma vez ou quatro vezes ao ano (Endress, Gorchov \& Noble, 2004; Endress et al., 2006). Menor sobrevida também esteve associada à coleta de compostos secundários, como resinas ou exudatos (Soehartono \& Newton, 2001; Varghese \& Ticktin, 2008) e raízes aéreas (Plowden et al., 2003).

Assim como a sistematização das evidências demonstrou, o argumento de que ocorre redução na sobrevida após a extração de partes vegetativas e compostos secundários é recorrente na literatura (e.g.,Peters, 1994; Walters, 1997; Cunningham, 2001). A sobrevivência é ameaçada, pois a extração de partes da planta pode torná-la suscetível a patógenos ou causar estresse ao expor tecidos caulinares, prejudicando o fluxo de água e de nutrientes (Cunningham, 2001). 
Todavia, ressalta-se que há estudos que não observaram alterações nas taxas de sobrevivência, ao menos no curto prazo (após um ano), com a coleta de folhas de várias espécies de palmeiras de florestas neotropicais úmidas ou secas (Zuidema et al., 2007; Calvo-Irabién et al., 2009) e de caules ou dos escapos de duas espécies de savanas (Griffiths et al., 2005; Schmidt et al., 2007).

\subsubsection{Reprodução}

Em quinto lugar $(9 \%, n=168)$, há os efeitos da exploração sobre a reprodução das plantas exploradas, tais como alterações na produção de frutos. Dentre estas, $60 \%$ consistem em efeitos negativos e o restante de efeitos nulos (Figura 3). Porém, essas evidências restringem-se a efeitos indiretos da coleta de folhas e caules. Nos casos avaliados, foi observada redução do número médio de frutos em indivíduos explorados devido à retirada concomitante de ramos que também continham inflorescências ou frutos (Ghimire et al., 2005).

Os casos avaliados confirmam argumentos prévios de que parâmetros reprodutivos podem ser afetados tanto diretamente, pela exploração das próprias partes reprodutivas, quanto indiretamente, pela exploração de partes vegetativas, pois a extração reduz a disponibilidade de recursos energéticos para reprodução (Cunningham, 2001). É o caso ao menos com a coleta de frutos, folhas e cascas, inexistindo evidências para compostos secundários. Ademais, há estudos em que não foram observadas alterações em parâmetros reprodutivos, como no caso das taxas de recrutamento após a coleta de $100 \%$ dos caules subterrâneos (rizomas), espécie em que restos deixados no solo são capazes de rebrotar (Ghimire et al., 2005). Os efeitos no recrutamento foram também nulos com baixas taxas de extração, como a coleta de $0,26 \%$ a $1,64 \%$ do estoque total de cascas de árvores (Bitahiro et al., 2006).

Por fim, destaca-se que alguns estudos sugerem que mudanças na época de coleta ou a proteção dos indivíduos reprodutivos podem minimizar efeitos negativos na reprodução (Endress et al., 2006).

\subsubsection{Taxa de crescimento}

Das dez evidências de efeitos sobre a taxa de crescimento dos indivíduos de populações exploradas, metade está associada a efeitos negativos, $40 \%$ a efeitos nulos e um caso de efeito positivo (Figura 3). A taxa de crescimento foi reduzida com a coleta de partes da planta, como folhas de palmeiras (e.g., Flores \& Ashton, 2000; Endress et al., 2006) ou a casca de uma arbórea (Warburgia salutares (Bertol.f.) Chiov. ) (Botha et al., 2004). Portanto, corrobora-se hipótese prévia de que as taxas de crescimento podem ser reduzidas quando coletadas partes vegetativas ou compostos secundários, devido à diminuição do vigor dos espécimes explorados (Hall \& Bawa, 1993).

Embora evidências de efeitos negativos sejam mais comuns e concordem com argumentos prévios (e.g., Cunningham, 2001), com certos níveis ou formas de coleta é possível que as taxas de crescimento não sejam afetadas, ou possam inclusive aumentar. De fato, duas avaliações relativas à extração em taxas entre 0,2 e 1,6\% do total do estoque de cascas de espécies arbóreas não observaram alterações na taxa de crescimento três anos após cada coleta (Bitahiro et al., 2006). Ainda, a avaliação dos efeitos da coleta de caule de espécie arbustiva (Gaultheria shallon Pursh) mostrou que, após um ano, os espécimes explorados cresceram a taxas maiores do que espécimes intactos (Cocksedge \& Titus, 2006). Assim, os resultados indicam que, embora as taxas 
de crescimento sejam afetadas negativamente, certos níveis de extração podem ser compensados, ao menos para a coleta de partes vegetativas e certas espécies. Porém, coletas frequentes e em maiores taxas têm maiores chances de, no longo prazo, afetarem o crescimento.

\subsubsection{Estrutura genética, distribuição espacial e razão sexual}

Apenas uma evidência foi identificada de efeitos da exploração para cada um dos parâmetros: estrutura genética, distribuição espacial e razão sexual (Figura 3).

O estudo que avaliou as consequências genéticas na população foi realizado com a raiz do ginseng americano (Panax quinquefolius L.) e mostrou redução na diversidade genética com a exploração (Cruse-Sanders et al., 2005)2005. Concorda, assim, com hipótese prévia que sugeria que a coleta alteraria frequências gênicas da população (Peters, 1994), por exemplo, induzindo à diminuição do tamanho médio de frutos pela coleta de frutos maiores. Contudo, cabe ressaltar que há apenas uma evidência, tornando generalizações inadequadas.

No caso da distribuição espacial, argumenta-se que a coleta de PFNM pode transformar a distribuição espacial local da população explorada (Kusters, 2009). Porém, existe apenas o estudo de Muraleedharan (2005) que corroborou esta hipótese, ao observar que a distribuição espacial de espécies de PFNM coletadas em três áreas na Índia foi alterada por mudanças nos padrões de mortalidade e regeneração. $\mathrm{O}$ delineamento do estudo, todavia, não incluiu populações-controle e séries temporais, o que limita a confiabilidade da inferência.

Outro fator que poderia ser modificado pela coleta de PFNM é a razão sexual da população no caso de espécies dioicas (Kusters, 2009), importante pois altera o tamanho efetivo populacional e, sendo assim, a capacidade reprodutiva. Há apenas uma evidência desse efeito relativo à coleta de frutos da palmeira Mauritia flexuosa L.f. que, usualmente, ocorre por meio da derrubada da planta. Após a exploração, as populações das 12 localidades estudadas sofreram redução na proporção ente plantas fêmeas e machos (em média, 1 fêmea:3,5 machos), alterando as taxas de recrutamento de jovens com consequências demográficas indefinidas (Horn et al., 2012).

\subsection{Efeitos sobre a comunidade biótica}

Apenas dez casos avaliaram os efeitos da exploração de PFNM sobre a comunidade biótica, distribuídos entre os três parâmetros considerados: composição, interações planta-planta e planta-animal.

\subsubsection{Composição}

Os efeitos mais avaliados referem-se à composição de espécies da comunidade explorada $(63 \%, \mathrm{n}=$ 14), dentre os quais $90 \%$ observaram efeitos negativos e um caso não mostrou alterações (Figura 3).

Estudos relativos à coleta de frutos de diferentes espécies (e.g., Shankar et al., 1998; Moegenburg \& Levey, 2003), caules de Euterpe edulis Mart. (Chediack, 2008) ou musgos (Studlar \& Peck, 2007) observaram, assim como sugerido previamente (Ticktin, 2004), a redução na riqueza de espécies das localidades de extração. Todavia, estudo com coleta de briófitas não observou redução na riqueza ou alterações na composição de espécies da comunidade, ao menos quando a exploração ocorreu em parte da comunidade (padrão fragmentado), pois 
essa forma apenas acelerou o padrão natural de distúrbio. Padrões de extração que afetam toda a localidade podem causar efeitos maiores na comunidade (Peck \& Frelich, 2008), evidenciando que resguardar parte da população pode ser estratégia de manejo que reduz os efeitos negativos no restante da comunidade.

\subsubsection{Interações planta-animal}

Apenas três casos avaliaram os efeitos da coleta sobre as interações entre plantas e animais da comunidade onde ocorre a exploração (Figura 3). Corroborando a literatura prévia (Hall \& Bawa, 1993; Peters, 1994), uma destas evidências demonstrou que a extração produz efeitos negativos, como diminuição no número de visitas de aves e mamíferos frugívoros à área de coleta (Moegenburg \& Levey, 2003). Efeitos negativos também ocorreram com a coleta de sementes, pelo aumento da caça de animais dispersores, atividade que costuma acompanhar o extrativismo (Forget \& Jansen, 2007). Contudo, para a extração de compostos secundários, a única evidência existente apenas argumenta que a probabilidade da extração afetar outras espécies é pequena, sem na verdade levantar dados diretos neste sentido (Paoli et al., 2001). No caso, o resultado nulo ocorreria, pois a proporção de biomassa retirada da floresta e os métodos de coleta não alteravam condições locais. Ainda, e mais importante, a planta frutificava em períodos supra-anuais e em sincronia com a maioria das espécies de dossel, sugerindo pequena dependência deste recurso explorado por outras espécies. Embora estes resultados possam ser idiossincráticos, reforçam a importância de avaliar os métodos de coleta, as taxas de extração e a história natural da espécie explorada na análise dos efeitos do extrativismo.

\subsubsection{Interações planta-planta}

Há apenas uma evidência dos efeitos da coleta de PFNM nas interações com outras espécies vegetais da comunidade (Figura 3). Nesta, a coleta de E. edulis Mart. ocasionou mudanças nas relações de competição e dominância entre a espécie explorada e outras árvores e lianas da mesma localidade (Chediack, 2008), assim como proposto por Ticktin (2004).

\section{Conclusões}

Os resultados mostraram que o conhecimento sobre os efeitos ecológicos da extração de PFNM, apesar de crescente, ainda é limitado e não abarca todas as regiões do mundo, assim como todos os parâmetros biológicos importantes. Em sua maioria, os estudos avaliaram aspectos populacionais, com poucas evidências dos efeitos sobre a comunidade e nenhuma nas escalas de ecossistema e paisagem.

Apesar dessas limitações, pode-se afirmar que efeitos negativos na conservação do recurso explorado são recorrentes, em especial quando se coletam partes como folhas e cascas em grandes quantidades. A diversidade de evidências acumuladas em termos de espécies, grupos taxonômicos, hábitos, ecossistemas, técnicas e volumes de extração mostra que os efeitos negativos são provavelmente comuns e não particulares a certas espécies ou a condições ambientais específicas. Em particular, os resultados sugerem que os parâmetros mais frequentemente alterados pela extração de PFNM são: o tamanho populacional; as partes vegetais da planta explorada; a distribuição de classes de tamanho da população explorada; a probabilidade de sobrevivência das plantas das populações exploradas; e a riqueza de espécies na comunidade na qual a 
exploração ocorre. Portanto, apesar de a coleta de PFNM causar efeitos menores que outros usos da terra, ainda assim pode provocar efeitos ecológicos significativos e ser insustentável no longo prazo.
Evidencia-se, assim, a necessidade da elaboração e implementação de planos de monitoramento e de manejo dos PFNM quando a extração ocorre em ecossistemas naturais.

\section{Referências}

Akobeng, A. Understanding systematic reviews and metaanalysis. Archives of Disease in Childhood, 90, 845-848, 2005.

Avocèvou-Ayisso, C.; Sinsin, B.; Adégbi, A.; Dossou, G. et al. Sustainable use of non-timber forest products: impact of fruit harvesting on Pentadesma butyracea regeneration and financial analysis of its products trade in Benin. Forest Ecology and Management, 257, 1930-1938, 2009. doi: 10.1016/j.foreco.2009.01.043.

Bitahiro, R.; McNeilage, A.; Babaasa, D.; Barigyra, B. Plant harvest impacts and sustainability in Bwindi Impenetrable National Park, S.W. Uganda. African Journal of Ecology, 44, 14-21, 2006. doi: 10.1111/j.1365-2028.2006.00597.x.

Botha, J.; Witkowski, E. T. F.; Shackleton, C. M. The impact of commercial harvesting on Warbugia salutais (pepper-bark tree) in Mpumalanga, South Africa. Biodiversity and Conservation, 13, 1675-1698, 2004. doi: 10.1023/B:BIOC.0000029333.72945.b0.

Calvo-Irabién, L.; Zapata, M. T.; Iriarte-Vivar, S. Effects of leaf harvest on Thrinax radiata palm: implications for management and conservation. Journal of Tropical Forest Science, 21(1), 34-44, 2009.

Chediack, S. The effect of forest exploitation on structure, diversity, and floristic composition of palmito-dominated Atlantic forests at Misiones, Argentina. Revista de Biologia Tropical, 56(2), 721-738, 2008.

Chen, Y. Song rong (Tricholoma matsutake) a valuable forest mushroom from China: consumption, development and sustainability. In: Kusters, K.; Belcher, B. (Eds.). Forest products, livelihoods and conservation. Case studies of non-timber forest product systems. Bogor: CIFOR, 2004. p. 78-93.

Cocks, M.; Dold, T. The informal trade of Cassipourea flanaganii as a cosmetic in South Africa. In: Sunderland,
T.; Ndoye, N. (Eds.). Forest products, livelihoods and conservation. Case studies of non-timber forest product systems. Bogor: CIFOR, 2004. p. 73-90.

Cocksedge, W.; Titus, B. D. Short-term response of salal (Gaultheria shallon Pursh) to commercial harvesting for floral greenery. Agroforestry Systems, 68(2), 103-111, 2006. doi: 10.1007/s10457-006-9001-1.

Cruse-Sanders, J. M.; Hamrick, J. L.; Ahumada, J. A. Consequences of harvesting for genetic diversity in American ginseng (Panax quinquefolius L.): a simulation study. Biodivesity and Conservation, 14, 493-504, 2005. doi: 10.1007/ s10531-004-7308-7.

Cunningham, A. Applied ethnobotany: people, wild plant use and conservation. London: Earthscan Publications, 2001.

De Oliveira, R. L. C.; Neto, E. M. L.; Araújo, E. L.; Albuquerque, U. P. Conservation priorities and population structure of woody medicinal plants in an area of caatinga vegetation (Pernambuco State, NE Brazil). Environmental Monitorament and Assessment, 132, 189-206, 2007. doi: 10.1007/s10661-006-9528-7.

Delvaux, C. Recovery from bark harvesting of 12 medicinal tree species in Benin. West Africa. Journal of Applied Ecology, 46, 703-712, 2009. doi: 10.1111/j.13652664.2009.01639.x.

Egli, S.; Peter, M.; Buser, C.; Stahek, W. et al. Mushroom picking does not impair future harvests. Results of a long term study in Switzerland. Biological Conservation, 129, 271-276, 2006. doi: 10.1016/j.biocon.2005.10.042.

Emanuel, P.; Shackleton, C. M.; Baxter, J. S. Modelling the sustainable harvest of Sclerocarya birrea subsp. caffra fruits in the South African lowveld. Forest Ecology and Management, 214, 91-103, 2005. doi: 10.1016/j. foreco.2005.03.066. 
Endress, B. A.; Gorchov, D. L.; Berry, E. J. Sustainability of an non-timber forest product: effect of alternative leaf harvest practices over 6 years on yield and demography of the palm Chamaedorea radicalis. Forest Ecology and Management, 234, 181-191, 2006. doi: 10.1016/j. foreco.2006.07.020.

Endress, B. A.; Gorchov, D. L.; Noble, R. B. Non-timber product extraction: effects of harvest and browsing on an understory palm. Ecological Applications, 14(4), 11391153, 2004. doi: 10.1890/02-5365.

Endress, B. A.; Gorchov, D. L.; Peterson, M. B.; Serrano, E. P. Harvest of the palm Chamaedorea radicalis, its effects on leaf production, and implications for sustainable management. Conservation Biology, 18(3), 822-830, 2004. doi: 10.1111/j.1523-1739.2004.00073.x.

FAO. Global Forest Resources Assessment. Rome, Italy: Food and Agricultural Organization of the United Nations. 2005

Flores, C. F.; Ashton, P. M. Harvesting impact and economic value of Geonoma deversa, Arecaceae, an understory palm used for roof thatching in the Peruvian Amazon. Economic Botany, 54(3), 267-277, 2000. doi: 10.1007/BF02864781.

Forget, P. M.; Jansen, P. A. Hunting increases dispersal limitation into the tree Carapa procera, a nontimber forest product. Conservation Biology, 21(1), 106-113, 2007. doi: 10.1111/j.1523-1739.2006.00590.x.

Ganesan, R.; Setty, R. S. Regeneration of Amla, an important non-timber Forest product from Southern India. Conservation and Society, 2(2), 365-375, 2004.

Gaoue, O.; Ticktin. Impacts of bark and foliage harvest on Khaya senegalensis (Meliaceae) reproductive performance in Benin. Journal of Applied Ecology, 45, 34-40, 2008. doi: 10.1111/j.1365-2664.2007.01381.x.

Gebrehiwot, K.; Muys, B.; Haile, M.; Mitloehner, R. Introducing Boswellia papyrifera (Del.) Hochst and its non-timber Forest product, frankincense. International Forestry Review, 5(4), 348-353, 2003. doi: 10.1505/ IFOR.5.4.348.22661.

Ghimire, S. K.; McKey, D.; Aumeeruddy-Thomas, Y. Conservation of Himalayan medicinal plants: harvesting patterns and ecology of two threatened species, Nardostachys grandifolia DC. and Neopicroorhiza scrophulariiflora (Pennell) Hong. Biological Conservation, 124, 463-475, 2005. doi: 10.1016/j.biocon.2005.02.005.
Griffiths, A. D.; Schult, J. H.; Gorman, J. Wild harvest of Cycas arnhenica (Cycadaceae): impact on survival recruitment and growth in Arnhem land, northern Australia. Australian Journal of Botany, 53, 771-779, 2005. doi: 10.1071/BT04123.

Guedje, N.; Lejoly, J.; Nkongmeneck, B.-A.; Jonkers, W. B. J. Population dynamics of Garcinia lucida (Clusiaceae) in Cameroonian Atlantic forests. Forest Ecology and Management, 177, 231-24, 2003. doi: 10.1016/S03781127(02)00444-9.

Hall, P.; Bawa, K. Methods to assess the impact of the extraction of non-timber tropical forest products on plant populations. Economic Botany, 47(3), 234-24, 1993. doi: 10.1007/BF02862289.

Horn, C. M.; Gilmore, M. P.; Endress, B. A. Ecological and socio-economic factors influencing aguaje (Mauritia flexuo$s a)$ resource management in two indigenous communities in the Peruvian Amazon. Forest Ecology and Management, 267, 93-103, Mar 12012. doi: 10.1016/j.foreco.2011.11.040.

Kusters, K. Non-timber forest product trade: a trade-off between conservation and development. Assessing the outcomes of non-timber forest product trade on livelihoods and the environment, with special emphasis on the damar agroforests in Sumatra, Indonesia. Amsterdan, (Dissertation $\mathrm{PhD}$ ) - Universiteit van Amsterdan, 2009.

Kusters, K.; Achdiawan, R.; Belcher, B.; Ruiz, P., M. Balancing development and conservation? An assessment of livelihood and environmental outcomes of nontimber forest products in Asia, Africa, and Latin America. Ecology and Society, 11(20), 20, 2006.

Larsen, H. Commercial medicinal plant extraction in the hills of Nepal: local management system and ecological sustainability. Environmental Manegement, 29(1), 88-101, 2002. doi: 10.1007/s00267-001-0043-X.

Marshall, E.; Newton, A. C. Non-timber forest products in the community of El Terrero, Sierra de Manantlán Biosphere Reserve, Mexico: is their use sustainable? Economic Botany, 57(2), 262-278, 2003. doi: 10.1663/0013-0001(2003)057[0262:NFPITC]2.0.CO;2.

Moegenburg, S. M.; Levey, D. J. Do frugivores respond to fruit harvest? An experimental study of short term responses. Ecology and Society, 84(10), 2600-2612, 2003. doi: 10.1890/02-0063. 
Muraleedharan, P. e. a. Non-timber forest products in the Western Ghats of India: floristic attributes, extraction and regeneration. Journal of Tropical Forest Science, 17(12), 243-257, 2005.

Niels, A. P. R.; Martínez-Ramos, M.; Ackerly, D. D. Defoliation and growth in an understory palm: quantifying the contributions of compensatory responses. Ecology and Management, 84, 2905-2918, 2003. doi: 10.1890/02-0454.

Paoli, G. D.; Peart, D. R.; Leighton, M.; Samsoedin, I. An ecological and economic assessment of the non-timber forest product gaharu wood in Gunung Palung National Park, West Kalimantan, Indonesia. Conservation Biology, 15(6), 1721-1732, 2001. doi: 10.1046/j.1523-1739.2001.98586.x.

Peck, J. Regrowth of understory epiphytic bryophytes 10 years after simulated commercial moss harvest. Canadian Journal of Forest Resource, 36, 1749-1757, 2006. doi: 10.1139/x06-071.

Peck, J. E.; Frelich, L. E. Moss harvest truncates the successional development of epiphytic bryophytes in the Pacific northeast. Ecological Applications, 18(1), 146-158, 2008. doi: 10.1890/07-0145.1.

Pedersen, H. Production and harvest of fibers from Aphandra natalia (Palmae) in Ecuador. Forest Ecology and Management, 80, 155-161, 1996. doi: 10.1016/03781127(95)03632-6.

Peres, C. A.; Baider, C.; Zuidema, P. A.; Wadt, L. H. O. et al. Demographic threats to the sustainability of Brazil Nut exploitation. Science, 302(2112), 2003. doi: 10.1126/ science.1091698.

Peters, C. Sustainable harvest of non-timber plant resources in tropical moist forest: an ecological primer. Washington: WWF, 1994. p. 73.

Plowden, C.; Uhl, C.; Oliveira, F. A. The ecology and harvest potential of titica vine roots (Heteropsis flexuosa: Araceae) in the eastern Brazilian Amazon. Ecology and Management, 182, 59-73, 2003. doi: 10.1016/S03781127(03)00030-6.

Purvis, A.; Gittleman, J. L.; Cowlishaw, G.; Mace, G. M. Predicting extinction risk in declining species. Proceedings of the Royal Society B-Biological Sciences, 267(1456), 1947-1952, Oct2000. doi: 10.1098/rspb.2000.1234.
Raimondo, D. C.; Donaldson, J. S. Responses of cycads with different life histories to the impact of plant collecting: simulation models to determine important life history stages and population recovery times. Biological Conservation, 111, 345-358, 2003. doi: 10.1016/S0006-3207(02)00303-8.

Rock, J. H.; Beckage, B.; Gross, L. J. Population recovery following differential harvesting of Allium tricoccum Ait, in the Southern Appalachians. Biological Conservation, 116, 227-234, 2004. doi: 10.1016/S0006-3207(03)00193-9.

Rodriguéz-Buriticá, S.; Orjuela, M. A.; Galeano, G. Demography and life history of Geonoma orbignyana: an understory palm used as foliage in Colombia. Forest Ecology and Management, 211, 329-340, 2005. doi: 10.1016/j. foreco.2005.02.052.

Ros-Tonen, M. A. F.; Wiersum, K. F. The scope for improving rural livelihoods through non-timber forest products: An evolving research agenda. Forests, Trees and Livelihoods, 15(2), 129-148, 2005. doi: 10.1080/14728028.2005.9752516.

Ruiz-Pérez, M.; Belcher, B.; Achdiawan, R.; Alexiades, M. et al. Markets Drive the Specialization Strategies of Forest Peoples. Ecology \& Society, 9(2), 4 2004. Disponível em: $<$ http://www.ecologyandsociety.org/vol9/iss2/art4>.

Sanders, S.; McGraw, J. B. Harvest recovery of goldenseal, Hydrastis Canadensis L. American Midland Naturalist, 153, 87-94, 2005. doi: 10.1674/0003-0031(2005)153[0087:HR OGHC]2.0.CO;2.

Schmidt, I. B.; Figueiredo, I. B.; Scariot, A. Ethnobotany and effects of harvesting on the population ecology of Syngonanthus nitens (Bong.) Ruhland (Eriocaulaceae), a NTFP from Jalapão region, Central Brazil. Economic Botany, 61(1), 73-85, 2007. doi: 10.1663/0013-0001(2007)61[73:EAEO$\mathrm{HO}$ 2.0.CO;2.

Schmidt, I. B.; Mandle, L.; Ticktin, T.; Gaoue, O. G. What do matrix population models reveal about the sustainability of non-timber forest product harvest? Journal of Applied Ecology 48, 815-826, 2011. doi: 10.1111/j.13652664.2011.01999.x.

Shackleton, C.; Shackleton, S. The importance of nontimber forest products in rural livelihood security and as safety nets: a review of evidence from South Africa. South African Journal of Science, 100, 658-664, 2004. 
Shackleton, C.; Shackleton, S.; Shanley, P. Building a holistic picture: an integrative analysis of current and future prospects for non-timber forest products in a changing world. In: Shackleton, S.; Shackleton, C. et al (Eds.). Nontimber forest products in the global context. Heidelberg: Springer Berlin, v.7, 2011. p. 255-280.

Shackleton, C. M.; Guthrie, G.; Main, R. Estimating the potential role of commercial over-harvesting in resource viability: a case study of five useful tree species in South Africa. Land Degradation and Development, 16, 273-286, 2005. doi: 10.1002/ldr.652.

Shankar, U.; Murali, K. S.; Shaanker, R. U.; Ganeshaiah, K. N. et al. The extraction of non-timber forest products in the forests of Biligiri Rangan Hills, India. Impact on floristic diversity and population structure in a thorn scrub forest. Economic Botany, 52(3), 302-315, 1998. doi: 10.1007/BF02862149.

Siebert, S. Abundance and growth of Desmoncus orthacanthos Mart. (Palmae) in response to ligth and ramet harvesting in five Forest sites in Belize. Forest Ecology and Management, 137, 83-90, 2000. doi: 10.1016/S03781127(99)00316-3.

Sills, E.; Pattanayak, K.; Ferraro, J.; Alger, K. Abordagens analíticas na avaliação de impactos reais de programas de conservação. Megadiversidade, 12(1-2), 39-49, 2006.

Sinha, A.; Bawa, K. S. Harvesting techniques, hemiparasites and fruit production in two non-timber forest tree species in South India. Forest Ecology and Management, 168, 289300, 2002. doi: 10.1016/S0378-1127(01)00747-2.

Soehartono, T.; Newton, A. C. Conservation and sustainable use of tropical trees in the genus Aquilaria II. The impact of gaharu harvesting in Indonesia. Biological Conservation, 97, 29-41, 2001. doi: 10.1016/S0006-3207(00)00089-6.

Studlar, S. M.; Peck, J. E. Commercial moss harvest in the Appalachian Moutains of West Virginia: targed species and incidental take. The Bryologist, 110(4), 752-765, 2007. doi: 10.1639/0007-2745(2007)110[752:CMHITA]2.0.CO;2.

Ticktin, T. The ecological implications of harvesting nontimber forest products. Journal of Applied Ecology, 41, 11-21, 2004. doi: 10.1111/j.1365-2664.2004.00859.x.

Ticktin, T.; Shackleton, C. Harvesting Non-timber Forest Products Sustainability: Opportunities and Challenges. In:
Shackleton, S.;Shackleton, C. et al (Eds.). Non-Timber Forest Products in the Global Context. Berlin Heidelberg: Springer 2011. p.

Varghese, A.; Ticktin, T. Regional variation in non-timber forest product harvest strategies, trade, and ecological impacts: the case of black dammar (Canarium strictum Roxb.) use and conservation in the Nilgiri Biosphere Reserve, India. Ecology and Society, 13(2), 11, 2008. Disponível em: <http://www.ecologyandsociety.org/vol13/iss2/art11/>.

Vázquez-Lopez, J.; Vibrans, H.; García-Moya, E.; ValdezHernández, J. I. et al. Effects of harvesting on the structure of a neotropical woody bamboo (Otatea: Guaduinae) populations. Interciencia, 29(4), 201-211, 2004.

Wadt, L. H. O.; Kainer, K. A.; Staudhammer, C. L.; Serrano, R. O. P. Sustainable forest use in Brazilian extractive reserves: natural regeneration of brazil nut in exploited populations. Biological Conservation, 141, 332-346, 2008. doi: 10.1016/j.biocon.2007.10.007.

Walters, C. Challenges in adptive management of riparian and coastal ecosystems. Ecology and Society, 1(2), 1, 1997. Disponível em: $<\mathrm{http}: / / \mathrm{www}$. consecol.org/vol1/iss2/art1/>.

Widayati, A.; Carlisle, B. Impacts of rattan cane harvesting on vegetation structure and tree diversity of Conservation Forest in Buton, Indonesia. Forest Ecology and Management, 266, 206-215, 2012. doi: 10.1016/j. foreco.2011.11.018.

Zuidema, P. A.; Boot, R. G. A. Demography of Brazil nut tree (Bertholletia excelsa) in the Bolivian Amazon: impact of seed extraction on recruitment and population dynamics. Journal of Tropical Ecology, 18, 1-31, 2002. doi: 10.1017/ S0266467402002018.

Zuidema, P. A.; Boot, R. G. A. Demography of th Brazil nut tree (Bertholletia excelsa) in the Bolivian Amazon: impact of seed extraction on recruitment and population dynamics. Journal of Tropical Ecology, 18, 1-31, 2002.

Zuidema, P. A.; Kroon, H.; Werger, M. Testing sustainability by prospective and retrospective demographic analyses: evaluation form palm leaf harvest. Ecological Applications, 17(1), 118-128, 2007. doi: 10.1890/1051-0761(2007)017[0118:TSBPAR]2.0.CO;2. 